\title{
CORRESPONDENCE
}

\section{Is comorbidity equivalent to symptoms in asymptomatic AS?}

\author{
Daniyar Gilmanov, Annamaria Mazzone, Sergio Berti and Mattia Glauber
}

In their Perspectives article, Vaishnava et al. (Vaishnava, P., Fuster, V., Goldman, M. \& Bonow, R. O. Surgery for asymptomatic degenerative aortic and mitral valve disease. Nat. Rev. Cardiol. 8, 173-177 [2011]) $)^{1}$ suggest that early pre-emptive intervention is warranted in selected, asymptomatic individuals with aortic stenosis (AS). Other researchers have concluded that, in elderly patients with AS, a careful search for comorbidities and coexisting cardiac diseases appeared relevant for accurate risk stratification and the choice of therapeutic strategy. ${ }^{2}$ Although the proportion of asymptomatic patients in the series of Faggiano et al. was small (5.8\%), we would like to draw attention to this great clinical challenge in the management and clinical decision-making process, ${ }^{3-8}$ because surgery for asymptomatic patients with AS is still controversial.

Current indications for aortic valve replacement (AVR) relate particularly to symptom development, but symptoms are typically insidious in onset and are masked by adjustments in lifestyle and a reduction in physical activity to avoid these manifestations. Many patients are unaware of subtle changes in effort tolerance (under-reporting of symptoms), and symptoms can be camouflaged by comorbidities such as pulmonary disease, obesity, peripheral artery disease and subsequent claudication, musculoskeletal degenerative diseases, the residual consequences of ischemic neurological events, poor eyesight or blindness, and physical deconditioning. Not surprisingly, these pathologies are common in the elderly. Thus, clinical evaluation of AS in the presence of heterogeneous associated diseases is rendered particularly complex. Serum B-type natriuretic peptide (BNP) levels have been demonstrated to predict symptom onset in asymptomatic adults with AS. ${ }^{9}$ As a sensitive marker of cardiovascular failure, BNP level is indicative of cardiovascular dysfunction, particularly left ventricular overload, even in the absence of symptoms. The negative impact of comorbidities on circulation failure in AS suggests that BNP level could be a valuable biomarker criterion for clinical decision-making. Thus, when symptoms are not sufficient for diagnosis (the first-line diagnostic recommendation in current guidelines), this biochemical parameter can be used as an alternative. The 'watchful waiting' strategy of delaying surgery until symptoms develop ignores the long-term consequences of irreversible left-ventricular remodeling, which could potentially adversely affect perioperative and long-term outcomes. Systemic hypertension, coronary artery disease, lung disease, obesity, renal dysfunction, and other comorbidities are all confounding factors that can also alter cardiac structure and function, patient symptoms, and prognosis.

Like Vaishnava et al. we support a strategy of early surgical intervention before symptom onset and consider asymptomatic patients, especially individuals aged $>70-75$ years, with the above mentioned comorbidities as having 'symptoms' in the evaluation of indications for surgery with treatment algorithms described in international guidelines. Our data favor minimally invasive AVR in elderly patients (overall in-hospital mortality $1.8 \%$ in patients aged $>75$ years).${ }^{10}$ We consider this approach a first-line strategy in such patients. If the individual is extremely frail and can not support or survive even minimally invasive AVR with the necessity for extracorporeal circulation and aortic cross-clamping, the most bland (almost 'hemi-conservative') tactics of those available today-transcatheter aortic valve implantation-might be offered as a second-line, reserve strategy.
In conclusion, we agree with Vaishnava and colleagues that a systematic, multidisciplinary diagnostic approach is beneficial for patients with severe AS, in which degenerative features are associated with advanced age and copathologies. Integral (global, or all-embracing) risk estimation must be performed throughout by the multispecialist team, including careful discussion of operative risk and life expectancy, long-term morbidity and mortality and quality-of-life, treatment preferences, and the probability of reoperation. We even suggest that comorbidities in asymptomatic elderly patients could be considered to be 'symptom-equivalents' for prognostic stratification and therapeutic decision making, thanks to the widening spectrum of treatment options.

G. Pasquinucci Heart Hospital, 305, Via Aurelia Sud, loc. Montepepe, Massa, I-54100, Italy (D. Gilmanov, A. Mazzone, S. Berti, M. Glauber)

Correspondence to:

D. Gilmanov

drgilmanov@hotmail.it

\section{doi:10.1038/nrcardio.2011.203-c1}

Competing interests

The authors declare no competing interests.

\section{Contributions}

D. Gilmanov researched the data for the article, contributed to the discussion of content, wrote the article, and reviewed and edited the manuscript before submission. A. M. Mazzone, S. Berti, and M. Glauber contributed to the discussion of the content and to review and editing of the manuscript before submission.

1. Vaishnava, P., Fuster, V., Goldman, M. \& Bonow, R. O. Surgery for asymptomatic degenerative aortic and mitral valve disease. Nat. Rev. Cardiol. 8, 173-177 (2011).

2. Faggiano, P. et al. Prevalence of comorbidities and associated cardiac diseases in patients with valve aortic stenosis. Potential implications for the decision-making process. Int. J. Cardiol. doi:10.1016/j.ijcard.2011.02.026.

3. lung, B. \& Vahanian, A. Epidemiology of valvular heart disease in the adult. Nat. Rev. Cardiol. 8, 162-172 (2011).

4. lung, B. Management of asymptomatic aortic stenosis. Heart 97, 253-259 (2011).

5. Kaleschke, G. \& Baumgartner, H. Asymptomatic aortic stenosis: when to operate? Curr. Cardiol. Rep. 13, 220-225 (2011).

6. Vahanian, A. \& Otto, C. Risk stratification of patients with aortic stenosis. Eur. Heart J. 31, 416-423 (2010). 
7. Lancelotti, P. et al. Risk stratification in asymptomatic moderate to severe aortic stenosis: the importance of the valvular, arterial and ventricular interplay. Heart 96, 1364-1371 (2010).

8. Zamorano, J. L. \& Goncalves, A. Exercise echocardiography in aortic stenosis: one more piece in the puzzle. Eur. Heart J. 31, 1295-1297 (2010).

9. Pedrazzini, G. B. et al. Comparison of brain natriuretic peptide plasma levels versus logistic EuroSCORE in predicting in-hospital and late postoperative mortality in patients undergoing aortic valve replacement for symptomatic aortic stenosis. Am. J. Cardiol. 102, 749-754 (2008).

10. Miceli, A. et al. Minimally invasive aortic valve replacement as first choice in elderly patients: a propensity score analysis. Heart Surg. Forum 14 (Suppl. 2), S81-S82 (2011). 